\title{
La mondialisation de l'économie : de nouveaux enjeux, de nouveaux contextes culturels
}

\section{Olivier Sturge-Moore}

\section{(2) OpenEdition}

\section{Journals}

Édition électronique

URL : https://journals.openedition.org/asp/3182

DOI : 10.4000/asp.3182

ISSN : 2108-6354

\section{Éditeur}

Groupe d'étude et de recherche en anglais de spécialité

\section{Édition imprimée}

Date de publication : 1 décembre 1997

Pagination : 325-338

ISSN : 1246-8185

\section{Référence électronique}

Olivier Sturge-Moore, «La mondialisation de l'économie : de nouveaux enjeux, de nouveaux contextes culturels », ASp [En ligne], 15-18| 1997, mis en ligne le 13 novembre 2012, consulté le 21 septembre 2021. URL : http://journals.openedition.org/asp/3182 ; DOI : https://doi.org/10.4000/asp.3182

Ce document a été généré automatiquement le 21 septembre 2021.

Tous droits réservés 


\title{
La mondialisation de l'économie : de nouveaux enjeux, de nouveaux contextes culturels
}

\author{
Olivier Sturge-Moore
}

1 Il est certain que le processus engagé par la mondialisation de l'économie (globalisation en anglais), caractéristique des années 1980 et 1990, est maintenant irréversible. Il est cependant indéniable que cette évolution est loin d'être uniforme et que même si elle est nécessaire à une plus grande mobilité internationale des ressources, des échanges de marchandises, de services et de capitaux, elle engendre également de nouvelles formes d'exploitation, d'inégalités et d'injustices sociales. Nous savons par expérience que ces questions ne laisseront pas les étudiants indifférents : c'est le monde qu'ils auront à affronter, notamment dans leur vie professionnelle, de plus en plus tributaire de l'internationalisation.

2 Nous avons donc décidé de travailler sur cet aspect particulier de l'économie dans la mesure où il est indissociable de la notion de contexte culturel, ou plus précisément de l'évolution de la culture, de sa diversification, mais peut-être aussi de sa fragmentation. La nature des interdépendances entre les différentes cultures est maintenant remise en cause, ce qui a pour conséquence de fragiliser les cultures minoritaires à un point tel qu'elles risquent fort d'être emportées par le «raz-de-marée» mondialiste. Notre intention n'est pas de critiquer ce phénomène à la fois inévitable et nécessaire, mais également enthousiasmant à bien des égards. Nous voulons avant tout amener les étudiants à réfléchir objectivement sur les différentes conséquences de la mondialisation.

Cette idée nous est venue après la lecture du rapport de la Commission mondiale du développement culturel, intitulé Notre diversité créatrice, que l'UNESCO a publié (1995), sous la direction de Javier Pérez de Cuéllar, ancien Secrétaire de l'onu. Ce travail de trois années (1993-1995) est en fait la synthèse des travaux effectués dans le cadre de la décennie mondiale du développement culturel (1988-1997). Il ne s'agit pas à proprement parler d'un document de spécialité, mais les problèmes qu'il soulève sont eux, hautement 
spécialisés et représentent des enjeux de première importance pour l'avenir de la planète. Notre objectif dans cette communication n'est pas de traiter les aspects techniques de ces questions, mais d'étudier le rapport qui existe entre culture et développement économique.

J. Pérez de Cuéllar faisait à l'époque les observations suivantes :

On that occasion I observed that development efforts had often failed because the importance of the human factor -that complex web of relationships and beliefs, values and motivations, which lie at the very heart of a culture- had been underestimated in many development projects. $(1995: 7)$

5 L'aspect éthique de ces préoccupations ne laissera sûrement pas les étudiants indifférents :

Clearly, there was a need to transcend economics, without abandoning it. The notion of development itself had broadened, as people realized that economic criteria alone could not provide a programme for human dignity and well-being. (idem : 8)

6 L'idée d'entreprendre une étude corrélative de l'économique et du culturel à l'échelle mondiale devait bientôt se concrétiser (idem : 8-9) :

At its twenty-sixth session in 1991 the General Conference of UNEsco adopted a resolution requesting the Director-General, in co-operation with the SecretaryGeneral of the United Nations, to « establish an independent World Commission on Culture and Development comprising women and men drawn from all regions and eminent in diverse disciplines, to prepare a World Report on Culture and Development and proposals for both urgent and long-term action to meet cultural needs in the context of development $\%$.

7 Les observations suivantes de F. Mayor, Directeur général de l'UNESCO, résument bien l'esprit dans lequel les travaux de la Commission devaient se développer :

La question de savoir comment les civilisations peuvent progresser grâce à une meilleure imbrication de la culture et de l'économie est aujourd'hui au coeur du développement culturel, aussi bien dans les pays industrialisés que dans les pays en développement. L'économie n'a aucun sens si elle ne contribue pas de façon dynamique à la culture tout en en bénéficiant. (Mayor 1989 : 324)

Revenons maintenant à nos préoccupations pédagogiques. Nous sommes persuadés que ce genre de document sera plus motivant pour les étudiants que la plupart des matériels pédagogiques habituellement utilisés en cours. L'utilisation de documents authentiques est de toute évidence souhaitable, mais c'est avant tout l'authenticité du contenu, c'est-àdire ce qu'il peut réellement évoquer et éveiller en eux au niveau de leur sensibilité qui les motivera de manière déterminante. Le problème, pour le concepteur, se situera bien évidemment au niveau de la didactisation de tels documents.

Mais quelles sont plus précisément les questions soulevées dans le rapport de l'UNEsco. Écoutons J. Pérez de Cuéllar à nouveau (1995 : 8) :

New questions needed to be asked and old ones posed anew. What are the cultural and socio-cultural factors that affect development? What is the cultural impact of social and economic development? How are cultures and models of development related to one another? How can valuable elements of a traditional culture be combined with modernization? What are the cultural dimensions of individual and collective well-being?

10 Nous sentons bien que dans la dichotomie culture-développement, la préoccupation première de J. Pérez de Cuéllar est de sauvegarder la culture. Nous pourrions en fait 
rajouter de manière plus directe : « la culture est-elle compatible avec la modernité ? » Ou même : « dans quelle mesure la culture est-elle menacée par l'économisme? ».

Notons que ces interrogations pourraient servir, non seulement de base de réflexion, mais également de sujets de dissertation pour les étudiants, pour autant qu'un travail de préparation ait été effectué, leur permettant à la fois de préciser la question et de contextualiser la réponse.

Terminons cette brève présentation de ce rapport de l'unESCO avec un commentaire de James Wolfensohn, qui participa à son élaboration. L'esprit dans lequel le rapport fut conçu y transparaît clairement :

This report reveals the many facets of the human and social dynamic that enriches our thinking on development. It reminds us that understanding the cultural contexts and values within which the men, women and children of the world live is critical, as we go beyond macroeconomics to shape our collective destiny.

Il existe de nombreux ouvrages et revues spécialisées sur la mondialisation. Sans aller jusqu'à une approche " économiste ", c'est-à-dire tendant à tout expliquer, y compris les idées, valeurs et même les sentiments par les seuls facteurs économiques, la plupart de ces documents, souvent très techniques, n'abordent pas la dimension culturelle de la mondialisation. Cette perspective suggère que l'économique est une cause plutôt qu'une conséquence de divers phénomènes culturels. Le monde moderne serait-il, dans ces caractéristiques culturelles, en train de s'inféoder de manière inéluctable à la «toutepuissance» de l'économique? Autant de questions qui, selon nous, intéresseront les étudiants.

L'objectif de cet exposé ne se situe donc pas dans une analyse purement économique du processus de mondialisation, mais à déterminer dans quelle mesure et de quelle manière ce phénomène va transformer la notion de contexte culturel.

Notons tout d'abord que si resituer les textes basés sur les grandes théories économiques du passé dans leur contexte historique vient tout naturellement à l'esprit, il n'en va pas de même pour le monde contemporain car les grandes mutations qui le caractérisent deviennent des réalités beaucoup plus rapidement que par le passé.

Notons au passage que les plus brillants intellectuels, politologues et économistes se sont souvent trompés dans leurs prévisions ou se sont même laissés surprendre par des événements majeurs comme la chute du mur de Berlin ou le soudain démantèlement de l'Union Soviétique. Mais le monde contemporain, selon notre expérience, est celui qui préoccupe nos étudiants et nous avons remarqué que les discussions qui portent sur ces questions éveillent leur curiosité, tout en leur permettant d'aborder la terminologie économique. L'enseignant doit cependant toujours garder à l'esprit qu'il est indispensable qu'ils la réutilisent dans d'autres contextes.

D'une manière générale, les étudiants semblent manifester moins d'intérêt pour les textes économiques à caractère historique, aussi bien ceux qui concernent l'histoire des idées économiques que ceux relevant de l'histoire des faits économiques. L'étude de l'anglais est probablement ressentie par eux comme répondant, partiellement tout au moins, aux problèmes qui les préoccupent sur le plan pratique, notamment professionnel, plutôt que comme un moyen privilégié d'accéder à la connaissance d'une civilisation, ou même de la littérature des pays anglo-saxons, ce qui impliquerait nécessairement une approche historique. 
Si l'étude de la civilisation et de l'économie des pays anglo-saxons, notamment le Royaume-Uni et les États-Unisrestent une priorité, nous pensons qu'il serait souhaitable qu'elles soient orientées différemment de ce qui a été le cas jusqu'à présent, c'est-à-dire de manière moins dominante, voire ethnocentrique, "a monocultural global village ", pour reprendre l'expression de C. Kramsch (1987 in Kramsch 1993 : 235).

D'autre part, étant donné l'ampleur que prend le processus de mondialisation et la rapidité avec laquelle il se développe, il n'est plus possible aujourd'hui de se permettre "d'ignorer» l'existence de nombreux autres pays, acteurs à part entière (et parfois, victimes de la mondialisation). Il est bien connu que beaucoup de multinationales ont construit leur prospérité grâce à la main-d'œuvre bon marché des pays du «Tiers Monde» (terme d'ailleurs caduque depuis la chute du communisme). Les étudiants doivent être sensibilisés à ces nouveaux enjeux, qui s'expriment par de nouveaux contextes dans lesquels, les concepts d'interculturalité, d'altérité, d'acculturation, si présents et problématiques dans la didactique des langues, sont en train de prendre une portée nouvelle.

La mondialisation génère-t-elle plus de problèmes qu'elle n'en résout? Elle semble en tout cas soulever tant de questions qu'il est difficile, pour le moment, d'en évaluer les bienfaits. Il est de toute façon certain, que, sur le plan culturel et social tout au moins, nous sommes devant un paradoxe : d'un côté, une aspiration à une unité universelle et de l'autre un désir profond et légitime d'affirmation des identités culturelles, sans doute exacerbé parfois jusqu'à la violence, par le courant mondialiste, ressenti alors comme une menace.

21 Dans une interview accordée au Courrier de l'UNESCO (1996: 6), J. Pérez de Cuéllar déclarait :

Comment résoudre les paradoxes de la mondialisation? Comment trouver de l'harmonie et du sens au sein du nouveau désordre mondial, caractérisé par l'incertitude, l'incohérence et l'injustice ? Comment revendiquer son particularisme culturel tout en s'ouvrant aux perspectives des échanges planétaires ? Comment, en un mot, élaborer nos propres modèles de développement?

Et l'ancien Secrétaire général de l'onu poursuit ses réflexions, qui sonnent ici comme un véritable appel :

La mondialisation des marchés, de la technologie et de l'information fait craindre un nivellement qui favorise par contrecoup un processus de fragmentation accélérée. Plus les gens se rapprochent, plus ils se sentent isolés. Alors, il faut construire entre eux des passerelles. (idem : 6)

23 Cette référence à l'interculturel et aux problèmes qu'il pose invite le lecteur à réfléchir sur le respect de la différence, non seulement culturelle, mais également sur le plan économique :

Il y a nécessairement autant de modèles de développement que de cultures. (idem :

5)

La reconnaissance de l'interdépendance de ces deux paramètres doit mener à l'assujettissement de l'économie par la culture, et non l'inverse, ce qui est, à notre avis, l'un des grands dangers de la mondialisation.

Les nouveaux espoirs d'accroissement du niveau de vie à l'échelle mondiale s'accompagnent, à l'heure actuelle, d'autant de menaces de nouvelles formes d'injustices, d'inégalités, et qui sait, de nouveaux conflits, insoupçonnés jusqu'à maintenant. Autant de questions qui ne laisseront pas les étudiants indifférents. 
26 En ce qui concerne l'interculturel, qui a été l'objet d'une littérature de recherche très abondante, il doit être redéfini dans une perspective plus large, multiculturelle ou même pluriculturelle, c'est-à-dire plus respectueuse de la spécificité de chaque culture. Les contenus des méthodes de langue et autres documents pédagogiques ne peuvent plus, à l'heure actuelle, se permettre d'ignorer l'existence de ces mutations et de leurs enjeux, en se contentant, comme ils l'ont souvent fait dans le passé, de présenter un matériel uniquement fondé sur une « hégémonie » culturelle anglo-saxonne.

La mondialisation est-elle une nouvelle idéologie ou un état de fait? Le débat est largement ouvert, offrant la voie aux opinions les plus diverses. Toujours est-il que ce phénomène irréversible affecte, pour le meilleur et pour le pire, de nombreux aspects de la vie des hommes, notamment sur les plans économique, terminologique et culturel. Mais c'est peut-être sur ce dernier plan que les bouleversements seront les plus profonds, et probablement les plus difficiles à contrôler :

Nos sociétés sont prises dans un étrange cercle vicieux dont elles semblent ne pas avoir conscience : c'est au nom de la compétition internationale, et donc finalement au nom de l'indépendance des nations comme communautés historiques concrètes, qu'on leur demande de s'adapter aux conditions de l'économie moderne. Or [...] ce processus d'adaptation risque, si l'on n'y prend garde, de provoquer une destruction par l'intérieur de leur vitalité culturelle, qui seule finalement pourrait donner sens à la lutte. (Perret \& Roustang 1993 : 175-176)

En ce sens, la culture, en tant que fondement même de la société peut être considérée comme conditionnant entre autres choses, la forme que prend le développement économique. A. Sen, professeur d'économie et de philosophie à l'Université d'Harvard, attribue à la culture un rôle déterminant (1996:13) :

C'est la culture qui détermine ce à quoi nous tenons vraiment. Rien ne peut justifier en dernière analyse l'importance accordée à la croissance économique, ou à tout autre objectif, indépendamment des valeurs qui nous tiennent à coeur, et qui sont le reflet de notre culture.

La non-prise en compte de la spécificité de chaque culture menace leur survie. Leur disparition serait une véritable tragédie pour le patrimoine culturel de l'humanité, sans parler du drame humain qui en résulterait inéluctablement. F. Mayor (1989 : 322) déplore la lenteur avec laquelle l'humanité prend conscience de la relation entre la notion d'identité culturelle et celle de développement :

C'est notre prise de conscience tardive de ce résultat tragique qui confère aujourd'hui plus d'importance, dans le débat sur le développement, aux valeurs traditionnelles et aux dimensions culturelles du développement. Une communauté humaine peut-elle cependant préserver les valeurs culturelles indispensables à son identité et à son intégrité tout en changeant de conditions sociales pour améliorer la qualité de vie de ses membres?

La revue Esprit a consacré une grande partie de l'une de ses parutions (1996:33-154) au thème $\mathrm{du}$ "Choc des cultures à l'heure de la mondialisation ». Voici l'une des questions qu'un journaliste de la revue pose à J.-F. Bayart, au sujet de la mondialisation et de la tradition, question formulée de manière assez radicale, sinon provocatrice (1996:69) :

Esprit - Pour se replacer sur le seul terrain économique, n'y a-t-il pas dans ce que vous évoquez la manifestation d'une résistance culturelle identitaire, qui précéderait le broyage final du culturel par l'économique? Il y aurait ainsi une dialectique entre la réappropriation et un mouvement de destruction lent, mais irréversible. Il y aurait donc, in fine, une déstructuration de ce qui permettait à des groupes sociaux de tenir ensemble. 
31 Il serait beaucoup trop long de citer toute la réponse de J.-F. Bayart et nous avons sélectionné ce passage qui nous paraît le plus pertinent pour notre sujet. Selon J.-F. Bayart le phénomène est naturel : "l'histoire de l'humanité est faite de processus de déstructuration et de recomposition » (idem : 69), mais, selon lui, c'est la régionalisation du système international qui l'emportera :

Il y aura évidemment une poursuite de la mondialisation économique, selon un processus conflictuel, avec les grands enjeux des relations économiques entre le Japon et les États-Unis, entre les États-Unis et l'Europe : il y aura, en somme, de grandes parties de bras de fer, avec des trous noirs (que se passera-t-il en Russie? En Afrique ?) Dans le domaine politique, on s'aperçoit que la dominante est plutôt à une nouvelle régionalisation du système international.

Nous voyons que la dure réalité économique de la «Triade » resitue le concept d'Union européenne dans une perspective mondiale, soulignant le fait que son unification n'est qu'une étape, complexe certes, mais déjà tournée vers l'intégration mondiale de ce continent.

Serons-nous ainsi amenés, ou même contraints, de "réinventer les différences » selon l'expression de J.-F. Bayart (1996: 55), non pas dans un sens négatif, mais de manière à ce que chacun " protège » son patrimoine culturel contre le « nivellement » (probablement par la base) qui risque fort de résulter de la mondialisation.

34 Ces nouvelles interdépendances sont en fait indissociables de cette "réinvention des différences » :

Ce mouvement de diffusion (d'un certain nombre de techniques ou de représentations culturelles) est indissociable de la réinvention de tous les messages qui circulent à l'échelle du globe, c'est ce que les anthropologues post-modernes nomment aujourd'hui la "réinvention de la différence", réinvention que l'on observe dans tous les domaines, et au 1er chef dans le domaine culturel. (idem : 55)

Le désir, souvent teinté d'inquiétude, de protéger sa propre culture, est parfois mal interprété, c'est-à-dire comme marquant une tendance à l'ethnocentrisme. Mais il peut parfaitement ne partir que d'une intention louable et n'exclut en rien le désir de faire partager cette culture, ainsi que de trouver un authentique intérêt ou même une joie véritable dans la découverte d'autres cultures.

Mais nous savons combien le culturel est chevillé à d'autres domaines, notamment le domaine économique. Pour J.-F. Bayart, c'est la culture elle-même qui le marque de son empreinte :

Nous savons en effet que les actions politiques ou économiques sont des actions culturelles: on ne peut faire de politique ou d'économie sans mettre en branle des représentations, des symbolisations et finalement toute une série de figures imaginaires qui relèvent de l'activité culturelle. (idem : 58-59)

Nous voyons clairement dans ce passage l'interdépendance de domaines différents. Il est certain que le processus de mondialisation ne peut que rendre de plus en plus complexe cette notion d'interdépendance. Il est toutefois à remarquer que la dimension culturelle peut, au sein d'une culture donnée, servir de lien entre ces différents domaines, dans la mesure où elle est indissociable de chacun d'entre eux. En ce sens, le culturel peut être considéré comme transdisciplinaire par définition, et doit de ce fait jouer un rôle-clé dans les nouvelles interdépendances générées par la mondialisation.

Il est d'autre part évident que cette vaste mutation n'en est qu'à ses débuts et l'expression « économie mondiale » est en réalité peu satisfaisante car peu représentative de la réalité 
si l'on pense à un certain nombre de pays du Tiers-Monde dont le niveau de développement non seulement ne leur permet pas de participer et de bénéficier de cette évolution, mais les positionne, par voie de conséquences, en laissés pour compte du développement. F. Braudel (1972) proposait cette distinction:

Par économie mondiale s'entend l'économie du monde pris en son entier [...] Par économie-monde, mot que j'ai forgé à partir du mot allemand "Weltwirtschaft ". J'entends l'économie d'une portion seulement de notre planète, dans la mesure où elle forme un tout économique. (In Dictionnaire de Citations Économiques, Hatier, $1985: 240$ )

Il semblerait donc que la mondialisation soit à l'origine d'un double paradoxe: fragilisation donc désir de sauvegarder les cultures minoritaires d'une part et souci d'une plus grande efficacité sur le plan économique d'autre part, alors que les faits prouvent en partie le contraire. Nous disons « en partie » car tout n'est pas négatif, bien entendu. Mais on est toutefois en droit de se demander si, à cause de l'intense concurrence internationale à laquelle tous les secteurs de l'économie sont maintenant exposés, la mondialisation, par rapport à son évolution désordonnée, répond réellement aux besoins de tous, ou tout au moins du plus grand nombre, sans parler des problèmes écologiques qu'elle engendre. Personne ne songerait aujourd'hui à remettre en question le fait qu'elle est inévitable, ce qui serait aller dans le sens inverse de l'histoire. Mais les nations sontelles toutes véritablement prêtes, pour des raisons de stratégies commerciales, à reconnaître ses imperfections et chercher des solutions pour mieux la contrôler?

La mondialisation de l'économie vient ainsi considérablement compliquer la notion d'interculturalité, au travers de réseaux d'interdépendances plus denses, d'échanges beaucoup plus rapides et diversifiés, ayant pour conséquences des interactions plus complexes, exigeant davantage des participants sur le plan de leurs compétences sociolinguistiques.

41 Mais si une certaine uniformisation due à la mondialisation nous parait regrettable, mais inévitable, l'uniformisation culturelle, qui risque fort d'en résulter, sera également difficile à éviter complètement, car elle soulève des questions probablement encore plus complexes et diversifiées que celles relevant du linguistique seul. Mais nous ne pouvons pas nous attarder sur ces questions de politique linguistique et culturelle car elles dépassent largement le cadre de cet exposé.

42 Sur le plan pédagogique, cette évolution sans cesse plus rapide, rend particulièrement délicate l'élaboration de contenus pédagogiques, d'autant plus que nous ne connaissons bien évidemment pas avec précision la situation professionnelle dans laquelle se trouveront les étudiants après leurs études :

Which world do we introduce to learners of English ? In the case of some narrowly focused courses in language for specific purposes we are able to make reasonable predictions about future uses of the language and prepare our students accordingly. But in general language courses we cannot know what the learners'future communicative activity will be, what schematic knowledge they will need to engage to effectively carry it out. (Widdowson, $1988: 19$ ).

43 Nous pensons que la distinction qu'établit $\mathrm{H}$. Widdowson entre langue générale et la langue de spécialité, sur ce point précis, n'est que partiellement justifiable dans la mesure où nous considérons que les langues de spécialité sont, comme la langue générale, mais dans une moindre mesure et de manière différente, dépendantes du contexte culturel. 
Nous pensons que la culture, prise au sens anthropologique du terme, a beaucoup plus d'influence sur la culture professionnelle et par voie de conséquence sur l'utilisation de la langue en milieu professionnel qu'on ne pourrait le supposer au premier abord. En ce sens, un enseignement prenant en compte le contexte culturel, s'il s'articule autour d'une solide documentation sur un secteur d'activité, une entreprise ou une situation plus précise, peut représenter une réelle sensibilisation à une culture professionnelle.

Mais il est certain que la mondialisation des échanges exige de chacun une sensibilisation à de nouvelles formes linguistiques et culturelles, le contexte interactionnel étant fortement conditionné par les caractéristiques du pays, de la culture ou même de chaque communauté de discours :

One has to view the world Englishes in terms of speech fellowships. The defining context and acceptance of linguistic innovations must be related to the pragmatic context of each speech fellowship. This is particularly important in the case of institutionalized varieties of English. (Kachru, $1988: 17$ ).

Un autre élément vient compliquer la tâche de l'enseignant: c'est ce que B. Kachru appelle les « intranational contexts » $(1988: 14)$ qui, selon lui, sont l'objet de nombreuses interrogations. B. Kachru déplore le manque de recherche et de matériel pédagogique prenant en compte des contextes locaux, "intranationaux", pour des raisons commerciales plus que pédagogiques:

It is sad that often these methods are promoted without much familiarity with the intranational contexts in which the non-native varieties of English are actually used. (idem : 17).

Mais si l'idée de prendre en compte la culture de chaque communauté semble séduisante, quelle réalité a-t-elle sur le plan pédagogique, notamment par rapport à l'accélération de la diversification des représentations culturelles dues à la mondialisation? Il n'est évidemment pas possible d'initier les étudiants à tous les contextes qui existent. Mais faut-il pour autant se contenter de modèles méthodologiques simplifiés, standardisés, «passe-partout », tout au moins par rapport à leur contenu culturel ?:

The methodological aspect becomes important since one has to raise questions about the universal validity claimed for language teaching or language learning methods. The present tendency that the Western methods should be sold to the nonnative users with an evangelical zeal is suspect. It is particularly suspect since commercialism and ethnocentricism seem to determine such hard sell. (idem : 17)

La question est délicate, car elle ne fait que pointer vers l'hégémonie mondiale de la langue anglaise. Cet ethnocentrisme ne serait-il pas, au niveau de la conception méthodologique, une conséquence du trop grand nombre de spécificités culturelles à prendre en compte ? Un contenu de nature ethnocentrique serait ainsi la résultante d'une pure commodité, commodité dont le côté commercial risque de se faire de plus en plus pressant en raison de la mondialisation des marchés. Mais même en considérant que cette tendance au niveau des contenus soit due plus aux contraintes commerciales qu'à une tendance fâcheuse, mais dans une certaine mesure naturelle, à l'ethnocentrisme, il reste cependant indéniable, à notre connaissance, que la plupart des contenus culturels sont à sens unique : l'étudiant, sous prétexte de la découverte d'une culture étrangère, apprend "ce qu'il n'est pas ", ce qui ne fait qu'accentuer la problématique de l'altérité. Il serait sûrement plus fécond à tous points de vue, que l'apprenant exprime également «ce qu'il est ", vis-à-vis de son identité culturelle, de manière à développer une authentique interculturalité, tout au moins dans un cadre pédagogique. Cette démarche permettrait, entre autres choses, d'éviter l'élaboration de stéréotypes, ayant pour origine une vision 
ethnocentrique du monde. Elle éviterait tout ce qui est à l'opposé du dialogisme: les comparaisons arbitraires entre cultures, les jugements de valeur et les critères normatifs (ma culture me semble normale, toutes les autres m'apparaissent comme différentes, voire bizarres). Cette approche permettrait au contraire d'optimiser les différences. G. Zarate (1986 : 36) attire cependant notre attention sur le fait que :

La moralisation dans ce domaine est certainement l'un des pièges les plus tentants, surtout dans un contexte scolaire. L'expression « dialogue des cultures », qui induit une relation non conflictuelle entre cultures différentes, ou des réflexions s'appuyant sur des formules du type " concilier la fidélité à soi et l'ouverture aux autres » expriment davantage une bonne volonté qu'une réalité scientifiquement possible.

G. Zarate estime d'ailleurs que, en ce qui concerne enseigner des valeurs relevant d'une "philosophie" interculturelle, le professeur de langue dispose d'une "marge de manoeuvre étroite ", d'autant plus que, comme le fait remarquer C. Kramsch (1993: 48) :

The dominant culture of the classroom is constantly contested, avoided, put in question, confronted with linguistically deviant "minority" cultures. Many learners resist the self-evident and invisible culture teachers try to impose.

Les apprenants ne sont pas les seuls responsables (idem : 94) :

[...] teachers seem to pull the brake at precisely those points in the give-and-take of the lesson that could allow for a discovery and discussion of individual and social meanings.

Mais quelles que soient les considérations éthiques qui entrent en jeu, il reste indéniable qu'un enseignement de langue prenant en compte le contexte culturel ne peut que faciliter l'intégration de la dimension interculturelle. Il est cependant à remarquer que la mondialisation de l'économie vient considérablement compliquer l'ensemble des notions que nous venons de voir. Mais c'est probablement au niveau du contexte interactionnel que ces difficultés sont le plus facilement repérables, notamment dans le cadre des entreprises multinationales.

51 Il est de toute façon indispensable que le contexte culturel soit défini, ou même redéfini dans certains cas plus complexes, si l'on veut éviter les échecs pragmatiques. C'est ce que C. Kramsch fait remarquer dans le passage suivant, à propos de ses propres étudiants :

Ultimately, they found, it is one's willingness to abandon one's context of reference at deeply embedded points of misunderstanding and instead redefine the context itself, rather than continue to embroider on the same one, that leads to satisfactory communication. (Kramsch 1993 : 58).

Revenons aux réflexions de B. Kachru sur l'anglais dans un contexte mondial et plus précisément sur la recherche dans le domaine des langues de spécialité (Kachru 1988: 15) :

In ESP-oriented research we find two problems : ethnocentricism in approach and neglect of intranational motivations for the uses of English. In functional terms, there is something wrong with such an approach. There is, of course, nothing wrong with high proficiency goals. But with such goals we unconsciously cultivate language attitudes which have psychological, social, and educational implications. And, in some cases, such goals take us away from linguistic and functional realism.

Ces observations doivent trouver un écho sur le plan didactique, mais il sera néanmoins difficile d'éviter le côté artificiel de la plupart des échanges se déroulant en cours de langue :

Lorsqu'un apprenant suit des cours de langue étrangère dans son propre pays, la situation est peu propice au développement de sa compétence sociolinguistique, il 
aura rarement l'occasion de vivre les blocages pragmatiques d'un échange. (Narcy

1990 : 93) cependant une dimension essentielle du dialogisme. Sans cette authenticité, les échecs pragmatiques risquent d'être fréquents, faute d'un repérage culturel suffisamment précis pour déterminer un choix linguistique approprié. Cette problématique nous paraît fondamentalement la même dans les langues de spécialité qu'en langue générale, même si les stratégies mises en oeuvre ainsi que les objectifs à atteindre peuvent être très différents. En ce sens, nous ne sommes pas tout à fait d'accord avec B. Kachru, qui suggère que dans le cas des langues de spécialité, les réussites pragmatiques devraient être plus courantes que dans la langue générale :

Pragmatic success, then, is determined by the context of encounter, and the participants in the encounter. We should, of course, expect maximal pragmatic success in "survival registers" or "survival ESP " : e.g. seaspeak, aviation, diplomacy, and so on. In registers of law or medicine, we must investigate the localized strategies of lexicalization, mixing, switching, and lectal change. (Chamberlain \& Baumgardner $1988:$ 15) favoriser les réussites pragmatiques, mais nous avons cependant observé que la dimension sociolinguistique des interactions - que la mondialisation des échanges culturels est en train de rendre de plus en plus complexe - demeure le principal obstacle aux réussites pragmatiques. Nous estimons en ce sens qu'une interaction qui s'inscrit dans le cadre des langues de spécialité, c'est-à-dire tributaire du contexte culturel, sera exposée, bien que dans une moindre mesure, aux mêmes risques d'échecs pragmatiques qu'en langue générale. été rassemblé dans un but pédagogique et non pas avec un objectif relevant du domaine des sciences économiques. Nous avons cherché à ce que ces réflexions constituent une base de travail pouvant mener à l'élaboration de matériels pédagogiques évitant une approche ethnocentrique c'est-à-dire s'articulant principalement, dans beaucoup de cas tout au moins, sur l'étude de la civilisation britannique et américaine, ou présentant la culture d'autres pays, mais considérée d'un point de vue essentiellement anglo-saxon. La mondialisation est en ce sens une excellente occasion de découvrir d'autres cultures.

En ce qui concerne l'enseignement des langues de spécialité, il peut permettre l'étude d'interactions se déroulant dans des milieux professionnels où l'interculturel joue un rôle de plus en plus important, notamment dans le cadre des «multinationales multiculturelles ».

Pragmatique et dialogisme se conjuguent donc, ou tout au moins devraient se conjuguer, non seulement dans la réalité du monde de l'entreprise et des échanges internationaux, mais également au niveau des possibles applications pédagogiques inspirées par ces nouveaux enjeux.

Nos réflexions nous ont cependant amené à penser qu'il était difficilement envisageable de considérer l'enseignement du contexte culturel comme un domaine ayant sa spécificité didactique. Nous considérons donc que s'il y a une spécificité dans cette approche, elle se situe dans un travail pédagogique en « amont ». Si ce point peut paraître "secondaire» à certains concepteurs et praticiens, nous sommes convaincus qu'il facilitera grandement leurs tâches à accomplir en " aval », c'est-à-dire " sur le terrain », 
et comme nous l'avons vu, mener à une meilleure performance pragmatique. Enseigner le contexte culturel ne constitue pas une fin en soi mais un moyen d'aborder plus efficacement l'étude des langues de spécialité, notamment par une sensibilisation à l'ensemble des données culturelles dans lesquelles s'inscrit un document de spécialité. Le rapport de l'unESCO sur la culture et le développement nous a semblé un bon exemple de document pouvant amener les étudiants à la prise de conscience d'une problématique abordée de façon générale, mais qui permet d'aborder par la suite des domaines beaucoup plus précis, mais ayant toujours un rapport avec des aspects culturels plus généraux.

Concluons en faisant remarquer que l'approche que nous préconisons dans le cas particulier de la mondialisation de l'économie doit avant tout être considérée comme une préparation à l'étude de la langue de spécialité de l'économie, démarche que nous aimerions développer jusqu'à constituer une véritable "propédeutique » à l'apprentissage d'autres domaines de spécialité.

\section{BIBLIOGRAPHIE}

Bayart, J-F. 1996. « Du culturalisme comme idéologie. Entretien avec J.-F. Bayart ». In « Le choc des cultures à l'heure de la mondialisation ", Esprit 220, 54-71.

Chamberlain, D. \& R. J. Baumgardner (dir.). 1988. ESP in the Classroom: Practice and Evaluation. Oxford : Modern English Publications in association with The British Council, Coll. ELT documents, 128.

Kachru Braj, B. 1988. « ESP and non-native varieties of English: towards a shift in paradigm ». In Chamberlain, D. \& R. J. Baumgardner (dir.), ESP in the Classroom: Practice and Evaluation. Oxford : Modern English Publications in association with The British Council. Coll. ELT documents 128, 9-28.

Kramsch C. 1993. Context and Culture in Language Teaching. Oxford : Oxford University Press.

Mayor, F. 1989. Les ressources humaines de la dimension culturelle. Paris : OCDE.

Narcy J.-P. 1990. Apprendre une langue étrangère. Didactique des langues : le cas de l'anglais. Paris : Éditions d'Organisation.

Pérez de Cuéllar, J. 1995. « Children and young people ». In Pérez de Cuéllar, J. et alii, Our creative diversity. Report of the World Commission on Culture and Development. Paris : UNESCO Publishing, 151-172.

Pérez de Cuéllar, J. 1996. « Notre diversité créatrice ». Le Courrier de l'UNESCO 4-7.

Perret, B. \& G. Roustang. 1993. L économie contre la société. Affronter la crise de l'intégration sociale et culturelle. Paris : Le Seuil.

Sen, A. 1996. « Une question de choix. La culture ne serait-elle qu'un accessoire du développement?». Le Courrier de l'UNESCO, 10-13. 
Widdowson, H. G. 1988. « Aspects of culture and language learning ». In Interkulturelle Kommunication und Fremdsprachenlernen/Culture and Language Learning / Communication interculturelle et apprentissage des langues, Triangle 7, 13-22.

Wolfensohn, J. 1995. Our Creative Diversity. Report of the World Commission on Culture and Development . Paris : UNESCO publishing, 7-13.

Zarate, G. 1986. Enseigner une culture étrangère. Paris : Hachette.

\section{RÉSUMÉS}

La mondialisation de l'économie est l'un des faits les plus marquants de cette fin de siècle. Elle affecte de très nombreux domaines, et tout particulièrement la dimension culturelle. Le monde de l'entreprise est directement concerné et de nombreuses données qui en relèvent vont être amenées à être redéfinies, formant de nouveaux contextes culturels dont l'exploitation pédagogique nous paraît aussi passionnante que nécessaire pour les futurs utilisateurs que sont nos étudiants. Cet article a pour principal objectif d'attirer l'attention sur le fait que la recherche en didactique des langues de spécialité doit prendre en compte les nouveaux contextes culturels qui sont en train d'émerger de ce processus, sinon elle risque de se poser en paradoxe par rapport à l'extension et au rôle de l'anglais en tant que langue universelle mais dont la vocation doit être également multiculturelle.

The globalisation of the economy is one of the most striking features of the end of the 20th century. Many fields are affected, in particular the cultural one. The corporate world is directly concerned and many parameters will have to be redefined, bringing about new cultural contexts that ought to be exciting as well as necessary to exploit pedagogically for the future users that our students are. The principal aim of this article is to draw attention to the fact that unless research in language teaching takes these new cultural contexts into account, a paradox may arise concerning the expansion and the role of English as a universal but also multicultural language.

\section{INDEX}

Mots-clés : développement culturel, développement économique, dialogisme, pragmatique

Keywords : cultural development, dialogism, economic development, pragmatics

\section{AUTEUR}

\section{OLIVIER STURGE-MOORE}

